\title{
Mass Spectrometric Investigation of the Thermal Decomposition of Polymers ${ }^{1}$
}

\begin{abstract}
By Leo A. Wall
The application of the mass spectrometer to the study of the thermal decomposition of polymers is described. The relationships between the structure of polymers and yield of monomers on decomposition are discussed. A quantitative treatment relating the compositions of certain coploymers and the yields of monomers is given. Data are presented showing the composition of the volatile hydrocarbons produced by the thermal decomposition of several vinyl and diene polymers.
\end{abstract}

\section{Introduction}

The use of pyrolysis as a tool for investigating the constitution of high polymers has been limited by the difficulty involved in the analysis of the decomposition products. One of the best known examples of its use is the study of natural rubber by Midgley and Henne [1]. ${ }^{2}$ These investigators subjected $200 \mathrm{lb}$ of natural crepe rubber to distillation in iron vessels in 16-lb batches at atmospheric pressure and $700^{\circ} \mathrm{C}$. Analysis of the products disclosed 10 percent of isoprene and 20 percent of dipentene. The theoretical aspects of certain types of polymer pyrolysis have been discussed by Rice and Rice [2].

The recent development of the mass spectrometer as an analytical instrument [3] for hydrocarbon mixtures opened new possibilities for the utilization of pyrolysis as a means for the study of complex molecular structures by the analysis of their characteristic products of decomposition. The technique used in the present investigation consisted in pyrolyzing the polymers under conditions similar to a single-stage molecular distillation. This procedure was adopted to facilitate the removal of primary products before secondary decomposition became appreciable. The fact that a very small sample, $0.001 \mathrm{~g}$, is required increased the utility of the method; it would perhaps be advantageous in the investigation of biological materials.

\footnotetext{
1 Supported in part by funds from Reconstruction Finance Corp., Office of Rubber Research.

${ }^{2}$ Figures in brackets indicate the literature references at the end of this paper
}

\section{Experimental Procedure}

The pyrolysis tube or single-stage molecular still (fig. 1) resembles a test tube with a small side arm. The section of $3-\mathrm{mm}$ tube sealed on the top of the tube fits into a break-off device on the spectrometer.

The samples were inserted either as small lumps or as solutions. The preferred method of inserting the sample was to pipette into the side arm 2 $\mathrm{ml}$ of a solution of the polymer in a volatile solvent at a concentration of about $0.0005 \mathrm{~g} / \mathrm{ml}$. Upon evaporation of solvent, the tube was sealed to a high vacuum system. After evacuation to a pressure of $10^{-5} \mathrm{~mm}$ of mercury, the still was sealed off. The volume of each still was $45 \mathrm{ml}$.

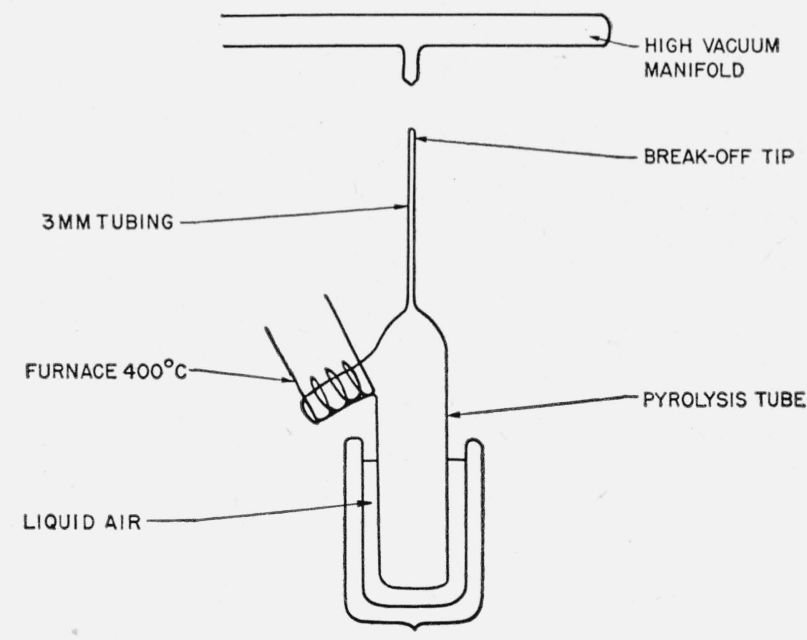

FIGURE 1. Diagram of pyrolysis apparatus. 
Pyrolysis was induced by heating electrically the side arm containing the polymer, and the body of the tube was immersed in liquid air to condense the products of decomposition. The time of heating was 20 minutes. A temperature of $400^{\circ} \mathrm{C}$ was used because it produced complete decomposition of most polymers, whereas lower temperatures did not.

After pyrolysis the tube was inserted in the mass spectrometer, the end of the small tubing was broken, and the volatile products were expanded directly into the inlet system (fig. 2). The total

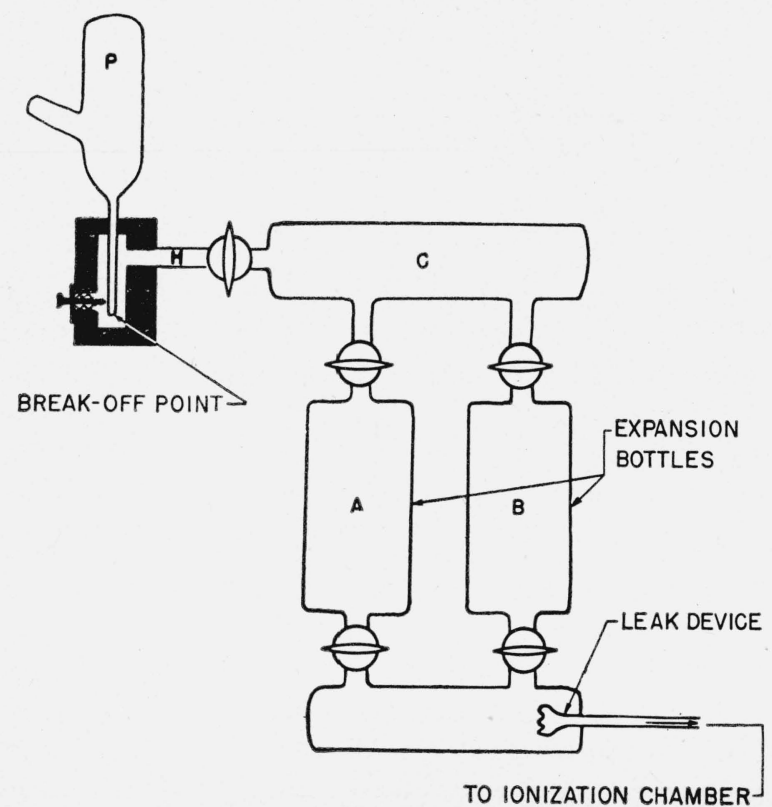

FIgure 2. Simplified diagram of mass spectrometer inlet system.

volume of the inlet system and the sample tube is known. Also, the partial pressure of each component can be determined by means of the known pattern sensitivities. The sensitivity for a compound is the height of a characteristic peak produced when 1-micron pressure of the pure substance is in the inlet system. The gas law is then used to compute the weight of the component produced, from which the percentage yield can be computed.

\section{Results of Tests}

Tables 1 and 2 show the various volatile hydrocarbons obtained in the pyrolysis experiments. As only products that have about $1 \mathrm{~mm}$ or more vapor pressure at room temperature could enter the Consolidated mass spectrometer, these results are given in terms of mole percent of "volatiles". The computed values, particularly where a large number of components occurred, are necessarily approximate. However, the uncertainty in the last significant figure is probably no more than 2 or 3 .

TABLE 1. Volatile products obtained in the pyrolysis of vinyl polymers

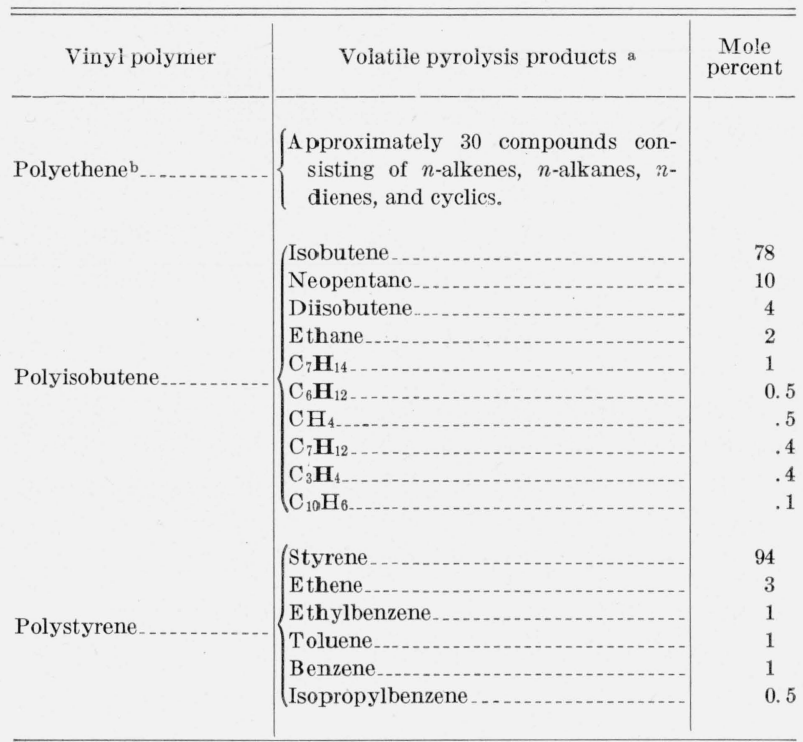

a The volatile products represent 66 percent of the polyisobutylene and 34 percent of the polystyrene.

b In polyethene, it proved impossible to compute an analysis since the products were so varied; however, the ethylene produced was certainly less than 1 percent.

The results vary considerably with the size of sample used. With $0.01 \mathrm{~g}$ the yield of isoprene from natural crepe was 18 percent, whereas with $0.001 \mathrm{~g}$ it was 2 percent. Apparently the $0.01-\mathrm{g}$ sample produced in the initial phase of the pyrolysis enough permanent gas to decrease the efficiency of the molecular distillation, thereby impeding the removal of the initial products. Dimers, trimers, etc., would thus be further cracked into monomers. More efficient molecular distillation would still further reduce the yield of monomer and produce chiefly degraded polymers within the molecular weight range of 100 to 1,000 . The latter figure corresponds approximately to the heaviest hydrocarbon species that can be distilled. Above this molecular weight, molecular cohesion is greater than the carbon-carbon single bond strength [4]. It can thus be seen that although low pressure decreases 
side reactions, monomer production is not necessarily improved, as dimers and larger molecules are formed and not subsequently decomposed.

Table 1 shows the analysis of the volatile

TABLE 2. Volatile products obtained in the pyrolysis of diene polymers

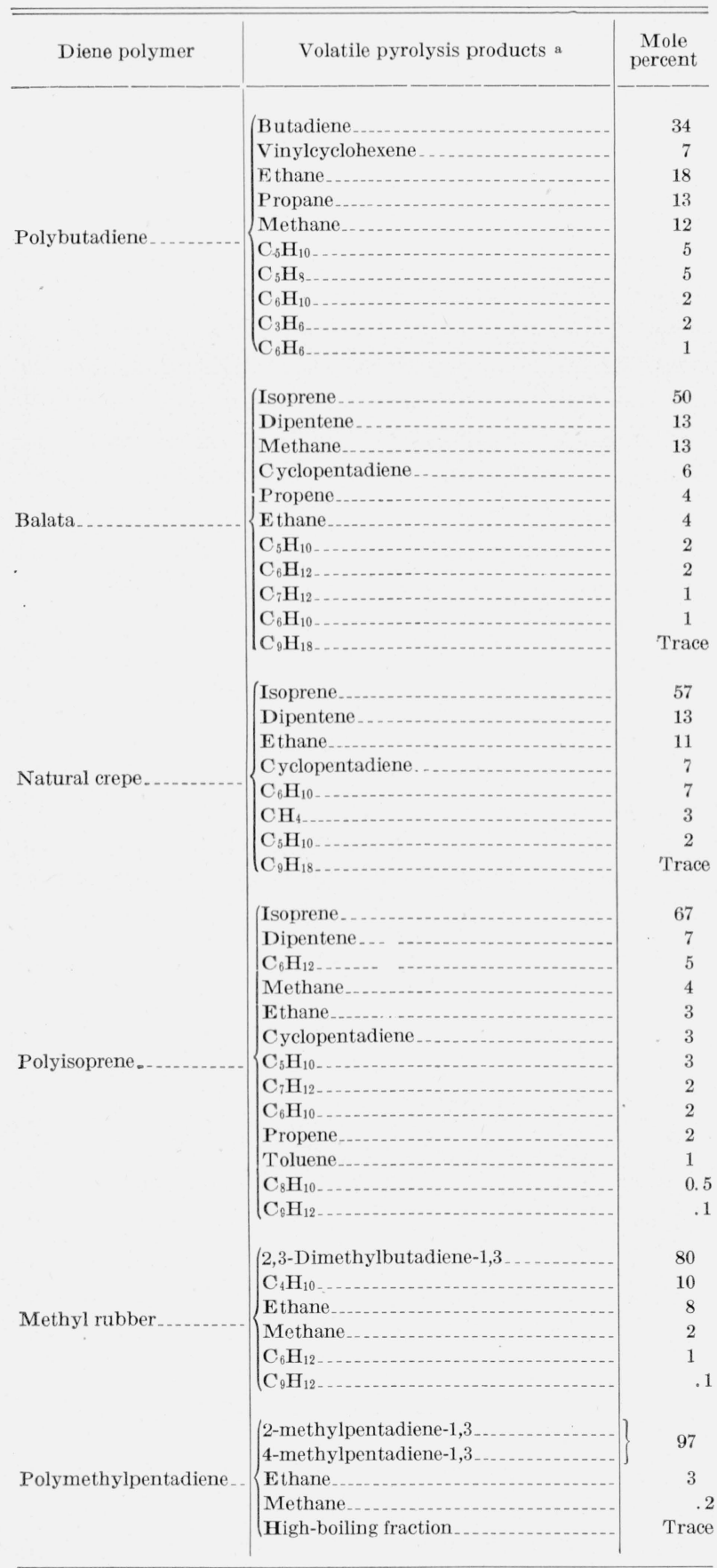

a The volatile products represent $3,4,4,8,7$, and 26 percent, respectively, of the polymers listed. products formed in the pyrolysis of vinyl polymers. The number and quantities of products other than monomer indicate the extent of various side reactions.

Table 2 presents the analysis of volatile products from some diene polymers. The three isoprene polymers are similar, and the poorer yield of monomer from the natural polymers may be due to the presence of impurities. The presence of cyclopentadiene in the products from the synthetic polymer would be expected, because it is usually present in commercial monomer; it was unexpected, however, in the case of the natural polymers.

Table 3 gives the monomer yields from the various polymers. In the case of three vinyl polymers, the yield is in the inverse order of their heat stabilities [5]. The heat stabilities of the diene polymers might therefore be expected to be in the inverse order of the monomer yields, with the exception of neoprene, which breaks down with the liberation of hydrogen chloride.

\section{TABLE 3. Monomer yields in pyrolysis of polymers}

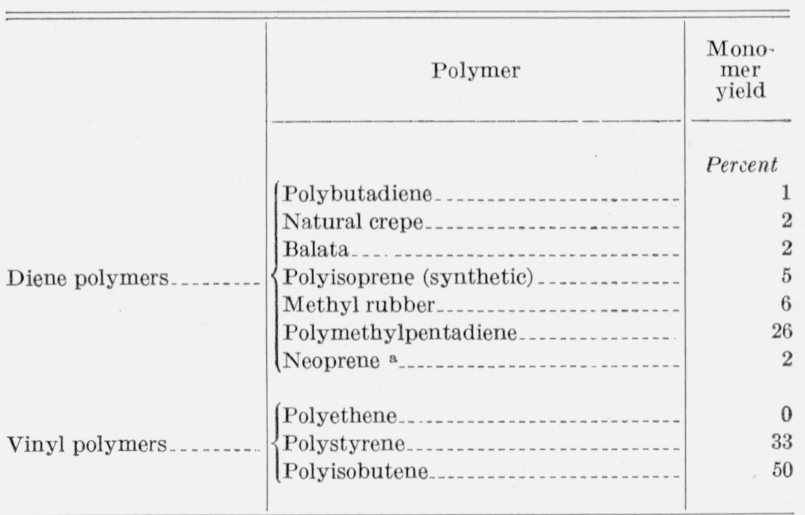

a The production of $\mathrm{HCl}$ accounted for 68 percent of the chlorine in necprene.

\section{Discussion of Results}

\section{Polymers From Single Monomers}

Thermal decomposition of polymers may be considered to occur through three types of reactions analogous to those leading to the formation of the polymer. The initial reaction is very likely the random breaking of some of the weakest bonds, which in hydrocarbons are the carboncarbon single bonds. A single break would form two radicals, which could easily disintegrate into small molecules and a small terminal radical. Other random breaks could form diradicals that 
could decompose only into small molecules, so that the over-all result would be similar to that when a single break is considered. Thus, the second type of reaction would be the formation of small molecules, sometimes chiefly monomer and terminal radicals. Finally, the small radicals would either acquire a hydrogen from other molecules, thus forming other radicals, or terminate by combining with each other or disproportionating. For high molecular-weight polymers, the quantity of products formed in the last step would be insignificant. However, if the large radicals prefer to pick off hydrogen atoms from other molecules thereby producing different radicals that are also capable of removing hydrogen or splitting into molecules and still other radicals, then there will be produced a variety of products other than monomer.

Whatever mechanism of thermal decomposition is considered, only products formed from the fragments of the chain ends would depend on the molecular weight and distribution. This effect would be appreciable only for rather low molecular weight polymers. Bachman et al. [6] report that the pyrolytic yield of styrene from low molecular weight polystyrene decreases with decreasing molecular weight of the polymer.

A simplified scheme for the thermal depolymerization of the vinyl polymers, except for the case where the $\mathrm{C}-\mathrm{Y}$ bond is weaker than the $\mathrm{C}-\mathrm{C}$ bond, may be postulated as follows:<smiles></smiles><smiles></smiles>

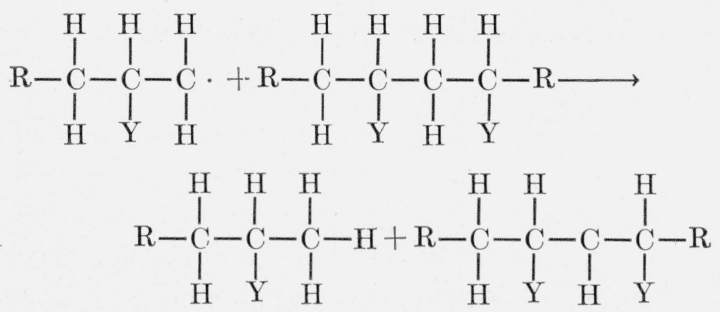

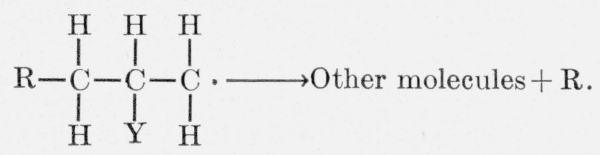

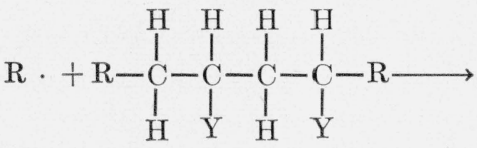

$$
\begin{aligned}
& \mathrm{R}-\mathrm{CH}=\mathrm{CHY}+\cdot \mathrm{CH}_{2} \mathrm{CHY}-\mathrm{R}+\mathrm{RH} \\
& 2 \mathrm{R} \cdot \longrightarrow \mathrm{R}-\mathrm{R}
\end{aligned}
$$

Hydrogen chloride is very easily stripped from polyvinyl chloride, so that the above reaction scheme is not applicable in this and similar cases, where the bonds to the substituted side groups are weaker than the $\mathrm{C}-\mathrm{C}$ bonds in the chain.

In a chain with head-to-tail structure, all of the $\mathrm{C}-\mathrm{C}$ bonds are identical, and even with other arrangements the bonds are certainly changed very little. With a head-to-tail structure, it can be seen by inspection that there are two ways of splitting out monomers. It can also be seen that headto-head and tail-to-tail structure decreases the number of ways and hence the probability of obtaining monomer. Therefore, the conditions favoring monomer are large molecular weight, head-to-tail structure, and a monomer that is both heat stable and unreactive. The complexity of the experimental results indicate that even under molecular distillation conditions the evaporation of products is slow compared to the reactions such as those postulated above. Most of the products obtained, however, can be formed as a consequence of the assumed scheme.

The relative rates of reaction $2 \mathrm{a}$ and $2 \mathrm{~b}$ should then determine the results of pyrolysis. Reaction $2 \mathrm{~b}$ depends on the reactivity of the radicals formed in the initial break. The activation engergies for the removal of a hydrogen by radicals should increase in the following order:<smiles>[R]C(C)C(=O)OC</smiles>

Kharasch [7] has found this order of reactivity for the removal of chlorine atoms from carbon tetrachloride. For the type of radicals on the right, there is no theoretical basis for readily predicting the exact order of reactivity; however, it is certain that they are quite unreactive compared to $\mathrm{RCH}_{2}$. Also, the radical $\mathrm{RCF}_{2}$ should have difficulty in removing fluorine from nearby chains. Polytetrafluoroethyelene [8], then, presumably de- 
composes chiefly in accordance with reaction $2 \mathrm{a}$. In general, all polymers giving appreciable yields of monomer on pyrolysis are capable of producing such relatively unreactive radicals through an initial scission of the chain. Since most of the common polymers are formed from mono or asymmetrically disubstituted monomers, the initial break produces two types of radicals, a reactive one and an unreactive one. The former rapidly picks up a hydrogen. It is then logical to conclude that the unreactive type of radical is the most abundant species of radical in the decomposing polymer and produces the major portion of monomer according to reaction $2 \mathrm{a}$.

Although radical reactivity seems to be the determining factor, it is linked with steric effects, since groups that stabilize the radical also cause increased steric hindrance. The bonds in the chain are consequently weaker, and decomposition will occur at lower temperatures. Thus reaction $2 \mathrm{a}$ is favored simultaneously by both steric and radical factors.

The results on polyisobutylene and polystyrene are interesting in this light. The styrene radical would ordinarily be assumed more unreactive than the tertiary butyl radical; however, polyisobutylene, in which there is known to be a large steric effect [9], produces the largest yield of monomer.

It is known that carbon-carbon bond strengths become weaker as one proceeds from primary to tertiary bonds [10]. This effect is probably independent of steric hindrance but not of the reactivity of the radicals formed by bond scission. Also, bonds adjacent or alpha to double and triple bonds are relatively stronger, whereas beta bonds are weaker.

A scheme similar to that for the vinyl decomposition can be postulated for the dienes. However, diene decomposition differs from that for the vinyls primarily because all of the bonds in the chain are not identical. Hence splitting will not be at random. The bonds in the beta position to the double bonds are presumed to be the weakest, and breaking of these bonds favors monomer production. Thus the effect on monomer yield of the weakness of these bonds in the dienes compensates for the dependence on the manner of splitting. Scission of any other bonds would lead to products other than monomer, dimer, etc., whereas in vinyls it does not matter which bond of the chain breaks first. In the diene case also, the radical reactivity effect appears to account for the differences found among such polymers. However, because of the double bonds in the chain, the dienes can react in many more ways than the vinyl polymers, and hence comparisons between the two types of polymers are not significant.

\section{Copolymers}

In all thermal decompositions there are competing reactions that the structure of the polymer either does or does not favor. This comes about through the operation of two effects: (1) probability, or the number of ways a certain result is obtained, and (2) bond strengths. The arrangement of substituent groups decides the first, and their nature decides the second. In copolymers, the study of these effects leads to some interesting conclusions.

For instance, in GR-S we have essentially styrene units isolated between butadiene units:

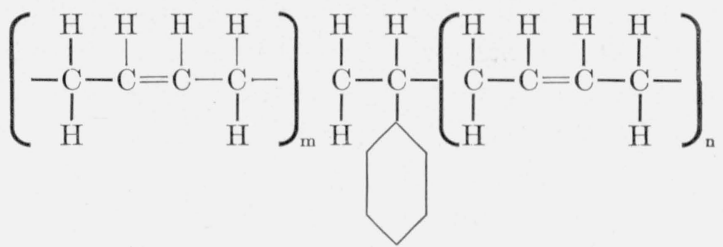

In this arrangement styrene can be obtained in only one way instead of the two possible in polystyrene. Hence the probability of obtaining styrene is halved. Theoretically, one should expect from GR-S, assuming that all styrene units were isolated, only half of the percentage of styrene yield possible on the basis of the polystyrene results. Thus, if by a given method the yield of styrene is 33 percent by weight from polystyrene, GR-S, which contains 23.5 percent by weight of styrene, should yield $23.5 \times 0.33 \times 1 / 2=3.9$ percent. The experimental yield was 3 percent.

In the case of the copolymer of methyl methacrylate with styrene, we find that, considering a head-to-tail structure and complete alternation,

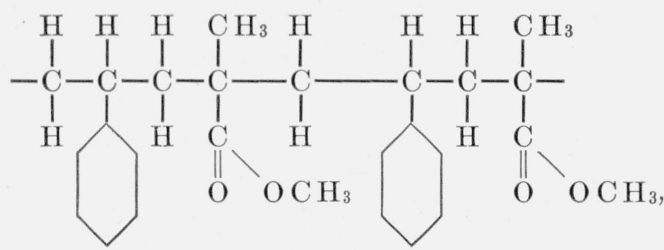

there are two ways of obtaining monomers. Hence, we would expect the same yields of mono- 
mer as from the simple polymers. This has been found to be true by Bachman, et al. [6], who recovered 66 percent of the styrene from such a copolymer, whereas under the same conditions polystyrene yielded 60 to 65 percent of its styrene. In the case of a symmetrical disubstituted ethene polymerized with a monosubstituted or asymmetrically disubstituted ethene, we should again have a copolymer that has only one way of splitting out monomer if the monomer units considered are isolated from one another.

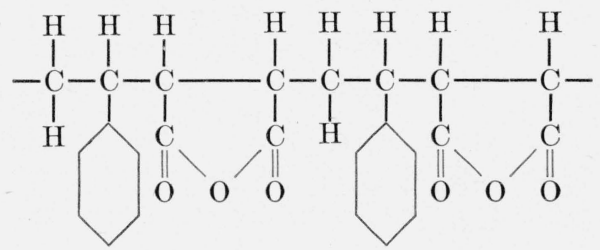

Bachman also depolymerized the copolymer of styrene and maleic anhydride. His yield would be expected to be $1 / 2 \times 60$, or 30 percent, of styrene recovered. Instead, he obtained 11 percent; however, the polymer loses carbon dioxide readily, so that side reactions may easily occur and thereby reduce the yield of styrene.

The above results confirm the assumed head-totail arrangement for polystyrene and polymethylmethacrylate. The low experimental yields from the copolymers imply an increased susceptibility to side reactions and cannot be attributed to head-to-head and tail-to-tail arrangements in the simple polymers.

Table 4 presents the comparative yields of styrene from various copolymers compared to the yield from polystyrene. It can be seen that the yield depends on the nature of the copolymer as well as the conditions of pyrolysis. In order to use pyrolysis as an analytical technique, one would

TABLE 4. Comparison of styrene yields from polymer and copolymers

\begin{tabular}{|c|c|c|}
\hline 1 & Polymer & $\begin{array}{l}\text { Styrene } \\
\text { yield a }\end{array}$ \\
\hline This work & $\left\{\begin{array}{l}\text { Polystyrene } \\
\text { GR-S }\end{array}\right.$ & $\begin{array}{r}\text { Percent } \\
33 \\
14\end{array}$ \\
\hline Bachman's work _. & $\left\{\begin{array}{l}\text { Polystyrene } \\
\text { Polystyrene-methylmethacrylate-...... } \\
\text { Polystyrene-maleicanhydride. }\end{array}\right.$ & $\begin{array}{r}60 \text { to } 65 \\
66 \\
11 \text { to } 12\end{array}$ \\
\hline
\end{tabular}

a Styrene yield $=\frac{\text { styrene recovered }}{\text { styrene in polymer }} 100$. need to know how the monomer units, were distributed in the copolymer which can be obtained from copolymer theory; or if one knew the amounts of different monomers in the polymer the yields would indicate their arrangement.

Assuming that the effect of side reactions on the yield of a given monomer remains constant in going from simple polymer to copolymers, and that the $\mathrm{C}-\mathrm{C}$ bonds in the vinyl chain and sequences are of equal strength for a given monomer, then the pyrolysis yield of monomer from the copolymer formed at low conversion can be calculated if one knows the yield from the simple polymer, the monomer reactivity ratios $\left(r_{A}\right.$ and $\left.r_{B}\right)[11,12]$, and the composition of the monomer charge. The probability of regenerating monomer $A$, for example, styrene, from the simple polymer can be considered unity. The probability of obtaining monomer $A$ from sequences $B A B$ consisting of say butadiene-styrene-butadiene units is $1 / 2$, from $B A A B$ sequences $3 / 4$, and from $B A_{1} A_{2} \ldots A_{i} B$ is $(2 i-1) / 2 i$.

It has been shown [13] that the probability of a given sequence containing $i$ monomer units is

$$
P_{B A} i_{B}=P_{B A} P_{A B} P_{A A}^{i-1},
$$

where $P_{B A}$ is the probability of a radical ending in monomer $B$ reacting with monomer $A$ in the process of copolymerization. Probabilities $P_{A B}$ and $\mathrm{P}_{A A}$ are similarly defined. They can be calculated from the following expressions:

$$
\begin{aligned}
P_{B A} & =\frac{A}{A+r_{B} B}, \\
P_{A B} & =\frac{B}{r_{A} A+B}, \\
P_{A A} & =\frac{r_{A} A}{r_{A} A+B},
\end{aligned}
$$

where $A$ and $B$ are the concentrations of the monomer in the mixture from which the polymer under consideration was formed.

The probability of obtaining monomer $A$ from any copolymer is simply the sum of the products of the probabilities of the various sequences and the probabilities of obtaining the monomer from the sequence. Hence

$$
\begin{aligned}
& P_{A} \text { from copolymer }= \\
& \sum_{i=1}^{i=\infty} \frac{2 i-1}{2 i} P_{B A} i_{B}=P_{B A B} \sum_{i=1}^{i=\infty} \frac{2 i-1}{2 i} P_{A A}^{i-1} .
\end{aligned}
$$


The yield of $A\left(Y_{A}\right)$ from copolymer would then be given by

$$
Y_{A}=N_{A} P_{B A B} \sum_{i=1}^{i=\infty} \frac{2 i-1}{2 i} P_{A A}^{i-1}
$$

or

$$
Y_{A}=N_{A}\left[P_{B A}+\frac{P_{B A B}}{2 P_{A A}} \ln P_{A B}\right]
$$

where $N_{A}$ is the yield of $A$ from its simple polymer under the same conditions used to decompose the copolymer of $A$ with $B$. By means of eq 5, 6 , and 7 , the function can be written in terms of monomer concentrations, $A$ and $B$, used in making the copolymer.

$$
Y_{A}=N_{A} \frac{A}{A+r_{B} B}\left[1+\frac{B}{2 r_{A} A} \ln \frac{B}{r_{A} A+B}\right]
$$

These equations are valid, of course, only for polymers formed at low degrees of conversions or where the relative monomer concentrations are constant during polymerization. Monomer $A$ can be any stable mono or asymmetrically disubstituted ethylene and $B$ any diene or symmetrically disubstituted ethylene. Deviations from the predicted results may be an indication of the extent to which the initial assumptions are not true.

Figure 3 is a plot of eq 11 applied to styrenebutadiene copolymer where $N_{A}$ is taken to be 33 . A 33-percent yield of styrene was obtained on the depolymerization of polystrene by our technique.

Figure 4 is a theoretical plot of yield against polymer composition. The straight line would

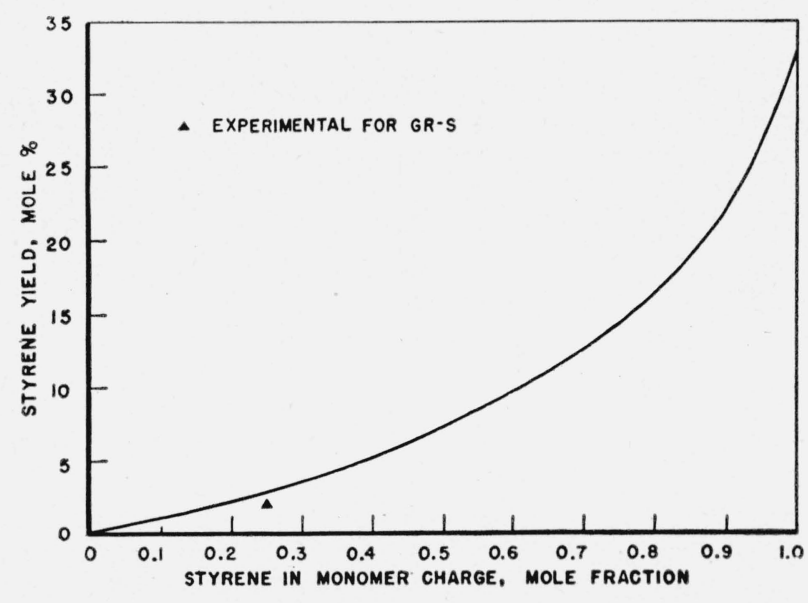

FiguRE 3. Styrene yield versus monomer composition.

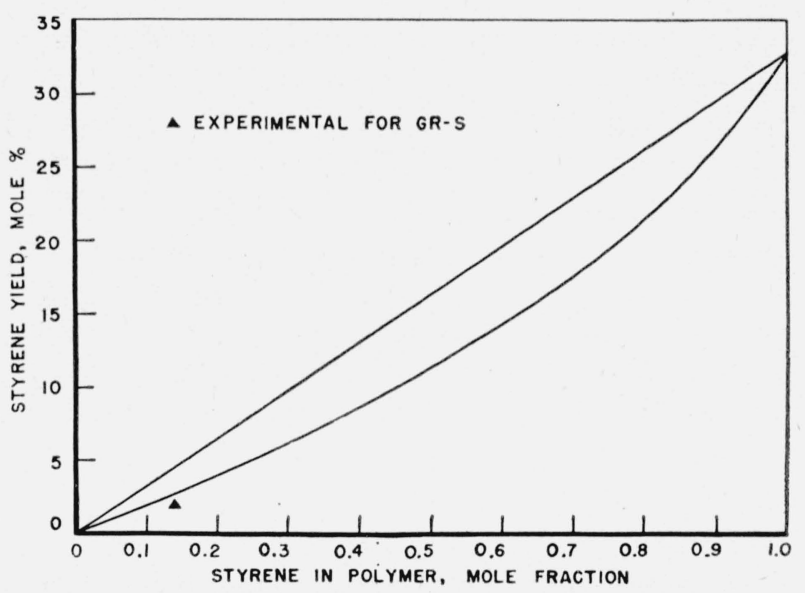

Figure 4. Styrene yield versus polymer composition.

apply if the copolymer were simply a mixture of polystyrene and polybutadiene, whereas the curved line would apply for a pure copolymer formed at low degrees of conversion.

The above-mentioned considerations, using monomer yield as a criterion for structural variations, should, of course, be correlated with yield of other products. Nevertheless, monomer yield alone can furnish information on the arrangement of the units, the number and types of sequences in certain copolymers, or composition. As the mass spectrometer is calibrated for more compounds, this technique should become increasingly valuable and perhaps become at least a supplementary analytical tool for the study of polymers.

\section{Conclusions}

The thermal decomposition. of polymers ean be studied by the use of the mass spectrometer to identify the volatile products. Small samples $(0.001 \mathrm{~g})$ are sufficient, and the experimental procedure is relatively simple. Qualitative estimates can be made rapidly, but precise analyses require considerable study of the mass spectrometric records. The chief limitations are that mass spectrometric data must be obtained for a large number of pure compounds, and that the studies are restricted to the volatile decomposition products; that is, those having a vapor pressure at room temperature of $1 \mathrm{~mm}$ of mercury or more.

Important structural arrangements in the polymer can be deduced from the nature of the 
pyrolysis products, but minor ones are frequently obscured because of the many secondary products formed. In general, it appears that monomers, from which relatively unreactive radicals are derived, have a higher degree of resonance stabilization and form polymers that decompose to a large extent into monomer. Polymers formed from such monomers appear more susceptible to thermal degradation. Polymers that have weakly held side groups, as well as fluorine substituted polymers, would form exceptions to this generalization.

Monomer yield alone can be used as a criterion of structure for certain copolymers and can be estimated from a knowledge of the relative reactivity of the monomers, the conditions of polymerization, and the results obtained with simple polymers.

The author thanks A. K. Brewer, F. O. Rice, and Robert Simha for their direction, encouragement, and suggestions. Acknowledgment is also made to Robert Reese, Lee Parham, and Vernon H. Dibeler for operating the mass spectrometer, and to Howard W. Bond for advice on problems of technique.

\section{References}

[1] T. Midgley and A. L. Henne, J. Am. Chem. Soc. 51, 1215 (1929).

[2] F. O. Rice and K. K. Rice, The aliphatic free radicals (Johns Hopkins Press, Baltimore, Md., 1935).

[3] H. W. Washburn, H. F. Wiley, and S. M. Rock, Ind. Eng. Chem., Anal. Ed. 15, 541 (1943).

[4] W. H. Carothers and J. W. Hill, J. Am. Chem. Soc. 54, 1557 (1932).

[5] R. B. Seymour, Ind. Eng. Chem. 40, 524 (1948).

[6] G. B. Bachman, H. Hellman, K. R. Robinson, R. W. Finhold, E. J. Kahler, L. J. Filar, L. V. Heisey, L. L. Lewis, and D. D. Micucci, J. Org. Chem. 12, 108 (1947).

[7] M. S. Kharasch, S. Kane, and H. C. Brown, J. Am. Chem. Soc. 64, 1621 (1942).

[8] E. E. Lewis and M. A. Naylor, J. Am. Chem. Soc. 69, 1968 (1947).

[9] A. G. Evans and M. Polanyi, J. Chem. Soc. 252 (1947).

[10] E. C. Baughan, M. G. Evans, and M. Polanyi, Trans. Faraday Soc. 37, 377 (1941).

[11] F. R. Mayo and F. M. Lewis, J. Am. Chem. Soc. 66, 1594 (1944).

[12] R. Simha and H. Branson, J. Chem. Phys. 12, 253 (1944).

[13] T. Alfrey and G. Goldfinger, J. Chem. Phys. 12, 205 (1944).

Washington, November 21, 1947. 